Suárez-Rodríguez, D.; Del Valle, M. (2019) Borg Scale and Intensity in Running and Specific Tennis Training. Revista Internacional de Medicina y Ciencias de la Actividad Física y el Deporte vol. 19 (75) pp. 399-413 $\mathrm{Http}: / /$ cdeporte.rediris.es/revista/revista75/artescala1035.htm

DOI: $10.15366 /$ rimcafd2019.75.002

\title{
ORIGINAL
}

\section{ESCALA DE BORG E INTENSIDAD EN ENTRENAMIENTOS DE CARRERA Y ESPECÍFICOS DE TENIS}

\section{BORG SCALE AND INTENSITY IN RUNNING AND SPECIFIC TENNIS TRAINING}

\author{
Suárez-Rodríguez, D. ${ }^{1}$ y Del Valle, M. ${ }^{2}$ \\ 1 Doctor en Ciencias del Deporte. Profesor de Ciencias del Deporte de la Universidad \\ Internacional Isabel I. Burgos (España) david.suarez9649@ui1.es \\ 2 Del Valle, Miguel. Doctor en Medicina. Director de la Escuela de Medicina del Deporte. \\ Universidad de Oviedo (España) miva@uniovi.es
}

AGRADECIMIENTOS: a Sixto Herrera García por su trabajo estadístico y a Alba García Celorio por su colaboración en la traducción.

Código UNESCO / UNESCO code: 2411 Fisiología Humana / Human physiology; 5899 Otras especialidades pedagógicas (Educación Física y Deporte) / Other specialties pedagogical (physical education and sport)

Clasificación del Consejo de Europa / Council of EuropeClassification: 4 Educación Física y deporte comparado / Physical education and sport compared; 6 Fisiología del Ejercicio / Exercise physiology

Recibido 4 de agosto de 2017 Received August 4, 2017

Aceptado 28 de noviembre de 2017 November November 28, 2017

\section{RESUMEN}

Se determinaron las diferencias en la intensidad y la valoración subjetiva de la fatiga en entrenamientos de carrera continua y específicos de tenis. Trece tenistas varones jóvenes tomaron parte en tres entrenamientos de resistencia de tipo continuo de 14 minutos de duración. Uno general consistente en carrera continua (CC), otro específico de peloteo cruzado (EP), y por último uno específico competitivo mediante puntos $(E C)$. Fue registrada la frecuencia cardiaca; y se valoró, al finalizar las tres pruebas, el lactato y la percepción de la fatiga. Se registraron valores más altos de frecuencia cardiaca y lactato en los dos ejercicios específicos, siendo significativamente superiores en EC respecto a CC (Frecuencia cardiaca $p>0.0054$. Lactato $p>0.0320$ ). Sin embargo, en la Escala de Borg no hubo diferencias significativas. Este estudio sugiere, para este 
grupo, que los valores de la Escala de Borg en ejercicios específicos de tenis pueden infravalorar la intensidad en relación con los de carrera.

PALABRAS CLAVE: Escala de Borg, frecuencia cardiaca, lactato, entrenamiento específico, tenis.

\begin{abstract}
Differences in intensity and subjective valuation of fatigue in continous running trainings and specific tennis exercises were determined. Thirteen young mal tennis players took part in three continuous type endurance training with 14 minutes length. A general one consisting of continuous running (CC) a specific one of a cross-knock up (EP) and finally a competitive specific one by points $(E C)$. The heart rate was recorded and the lactate and perception of fatigue were evaluated at the end of the three tests. Higher heart rate and lactate values were recorded in the two specific exercises being significantly higher in $E C$ regardin to $C C$ (Heart rate $p>0.0054$. Lactate $p>0.0320)$. However, there were no significant differences in the Borg Scale. This study suggest that the values of the Borg Scale in specific tennis exercises may undervalue the intensity in relation to the running values.
\end{abstract}

KEY WORDS: Borg Scale, heart rate, lactate, specific training, tennis.

\title{
INTRODUCCIÓN
}

La percepción del esfuerzo depende de la información de una serie de señales que proporcionan diversos receptores sensitivos. Esta percepción subjetiva resulta de gran interés por su relación con el estado anímico del jugador, pero al mismo tiempo por su relación con las adaptaciones vegetativas y metabólicas. Con el fin de valorarla se han creado diversas escalas, siendo la más utilizada la Escala de Borg. De esta Escala tenemos la original de 20 grados (Escala 20) y una modificada con una escala clásica numérica de 1 a 10 (Borg y Kaijser, 2006).

Se ha intentado establecer una relación entre la frecuencia cardiaca y los valores de la Escala de Borg (tabla 1), encontrándose en algunas investigaciones una alta correlación (Weston y col. 2006; Coquart y col. 2009; Sinclair y col. 2009). Sin bien, para alcanzar un alto grado de eficacia y precisión en la utilización de la Escala de Borg, como sistema de valoración de la intensidad del esfuerzo, resulta necesario un cierto periodo de familiarización (Faulkner y col., 2007).

Tabla 1: Relación entre el esfuerzo percibido (Escala de Borg), frecuencia cardiaca y porcentaje de la intensidad máxima. 


\begin{tabular}{|c|c|c|}
\hline Escala de Borg & Frecuencia cardiaca & $\%$ Intensidad \\
\hline 6 & $60-70$ & $10 \%$ \\
\hline 7 Muy, muy suave & $70-80$ & \\
\hline 8 & $80-100$ & $20 \%$ \\
\hline 9 Muy suave & $90-110$ & \\
\hline 10 & $100-120$ & $30 \%$ \\
\hline 11 Bastante suave & $110-130$ & \\
\hline 12 & $120-140$ & $40 \%$ \\
\hline 13 Algo duro & $130-150$ & $50 \%$ \\
\hline 14 & $140-160$ & $60 \%$ \\
\hline 15 Duro & $150-170$ & $70 \%$ \\
\hline 16 & $160-180$ & \\
\hline 17 Muy duro & $170-190$ & $80 \%$ \\
\hline 18 & $180-200$ & $90 \%$ \\
\hline 19 Muy, muy duro & $190-210$ & $100 \%$ \\
\hline 20 & $200-220$ & \\
\hline
\end{tabular}

Partiendo de estos resultados, algunos autores señalan su eficacia como herramienta para llevar a cabo un control continuo de las cargas más fácil y rápido, así como menos invasivo que el control de la frecuencia cardiaca, el lactato o la valoración del consumo de oxígeno de forma directa mediante espirométria portátil (Seiler y col., 2006; Cuadrado-Reyes y col., 2012), por lo que podría resultar por si sola muy útil para valorar los cambios en el nivel de entrenamiento.

Se está haciendo un gran uso en los deportes de equipo, tanto para controlar la carga durante los entrenamientos como en la competición (Alexiou y Coutts, 2008; Algroy y col. 2011; Calahorro y col. 2011; Cuadrado-Reyes y col. 2012). En tenistas se ha aplicado en algún estudio complementando la información obtenida mediante la frecuencia cardiaca y el lactato (Mendez-Villanueva y col. 2007).

A la hora de valorar la relación entre algunos parámetros fisiológicos y los psicológicos hay que conocer bien la respuesta al esfuerzo en cada deporte, y hacer una lectura compleja de los datos obtenidos. Así, en futbolistas, se ha podido observaren la segunda parte de los partidos (Torres-Luque y col., 2011), o en test específicos de alta intensidad (Ostojic y Mazic, 2002; Krustrup y col., 2006) resultados más altos en la Escala de Borg coincidiendo con una reducción en la producción de ácido láctico.

En los últimos años se vienen proponiendo, como forma especialmente idónea de entrenamiento, los ejercicios específicos. Sin embargo, es necesario conocer el comportamiento fisiológico y la percepción de la fatiga que se producen con este tipo de trabajos. Un factor que consideramos de gran interés es establecer las posibles diferencias, entre ejercicios generales y específicos en la valoración mediante la Escala de Borg. Nuestra hipótesis es que las diferencias entre ejercicios pueden modificarla percepción subjetiva de la fatiga y su relación con los parámetros fisiológicos.

\section{MATERIAL Y MÉTODO}


La muestra la integraron 13 tenistas varones. La edad media fue de $17 \pm 2$ años, la media de altura y de peso de $176.5 \pm 4.4 \mathrm{~cm}$ y $69.5 \pm 3.4 \mathrm{~kg}$ respectivamente. En el momento del estudio entrenaban $12 \pm 2 \mathrm{~h} /$ semanales y llevaban haciéndolo de forma regular $7 \pm 3$ años. Se trata de jugadores de nivel nacional, que preparaban la resistencia de forma habitual mediante la carrera y entrenamientos continuos e interválicos. Todos los sujetos fueron informados de las características del estudio, firmando su consentimiento previamente a la participación en el estudio.

\section{Protocolo experimental}

Antes de realizar el estudio se realizó una prueba de laboratorio consistente en un test de Conconi en cinta ergométrica, con el objeto de obtener la frecuencia cardiaca máxima individual de los jugadores.

El estudio experimental se realizó en el Centro Regional de Deportes del Principado de Asturias. Los ejercicios de carrera fueron realizados en un campo de futbol de hierba natural y los específicos de tenis en dos pistas de tenis cubiertas con una superficie de resina sintética de velocidad media.

Tres protocolos de entrenamiento:
a- Entrenamiento General de Carrera Continua (CC)
b- Entrenamiento Específico de Peloteo (EP).
c- Entrenamiento Específico Competitivo (EC).

Se realizó el estudio durante dos semanas: primero el trabajo de carrera continua, después el de peloteo y por último el específico competitivo. Cada tenista realizó dos pruebas con un tiempo de recuperación entre ellas de 72 horas.

CC. 14 minutos de carrera contínua con una intensidad al 90\% determinada por el propio sujeto. Se trata de un tiempo de trabajo óptimo para alcanzar intensidades elevadas así como para la mejora del consumo máximo de oxígeno (Morton y Billat, 1999).

EP. 14 minutos de peloteo cruzado por parejas con una intensidad del $90 \%$ determinada por los propios jugadores. El entrenador ponía la bola en juego nada más cometerse un error de forma que se mantuviera la continuidad del ejercicio.

EC. 14 minutos de puntos por parejas en toda la pista. La bola la ponía en juego el entrenador siendo el jugador que ganaba el punto el que golpeaba la primera bola en cruzado. La intensidad dependía de las características del juego competitivo.

Se realizó un calentamiento mediante 10 minutos de carrera suave previo al ejercicio de carrera (CC), y de 10 minutos de peloteo suave antes de los dos ejercicios específicos (EP y EC). 
Se registró la frecuencia cardiaca durante todas las pruebas con monitores de frecuencia cardiaca Polar (Polar RS400, Finlandia). Se midieron los niveles de lactato mediante analizadores portátiles Lactate Pro 2 (Arkray, Japón). Se realizaron las lactacidemias al final de cada prueba, mediante tomas de un volumenaproximado de $0.3 \mu \mathrm{l}$. Se solicitó también a cada sujeto, al final de los ejercicios la valoración de la fatiga mediante la Escala de Borg (Escala 20). Todos los datos fueron posteriormente organizados y analizados.

En el estudio estadístico se realizó una comparación de medias de las dos muestras mediante el T-test de dos colas que analiza la hipótesis de que las medias comparadas sean iguales frente a la alternativa de que no lo sean. En el test de hipótesis con un p-valor de 0.05 o inferior se alcanzará un nivel de significancia del $95 \%$. Se ha evaluado la normalidad de todas las muestras a través del test de Kolmogorov-Smirnov obteniendo que todas ellas son normales al $95 \%$ de significancia. Se aplica el T-test por diferentes razones: la primera porque todas las distribuciones son normales; la segunda porque la varianza de las muestras comparadas es igual, 0 al menos no difieren de forma estadísticamente significativa al $95 \%$ según el F-test de comparación de varianzas; finalmente, en virtud del tamaño muestral.

\section{RESULTADOS}

\section{Frecuencia cardiaca}

Se observan unos valores superiores en la frecuencia cardiaca en los esfuerzos específicos (tabla 2). La diferencia resulta significativa entre EC y CC (EC>CC) tanto en valores absolutos ( $p>\mathbf{0 . 0 0 5 4}$ ) como cuando se toman los datos relativos respecto a la frecuencia cardiaca máxima individual ( $p>0.0108)$.

Tabla 2: Valores medios y porcentaje respecto a la frecuencia máxima individual en esfuerzos generales de carrera y específicos de tenis. CC: Continuo Carrera. EP: Específico Peloteo. EC:

Específico Competición.

Diferencia significativa valores absolutos. EC $>$ CC. $p>0.0054$.

Diferencia significativa relativo a la frecuencia cardiaca máxima. $E C>C C, p>0.0108$.

\begin{tabular}{|l|c|c|c|}
\hline & CC & EP & EC \\
\hline FC media / Porcentaje & $175.08 / 89.08 \%$ & $177.54 / 90.33 \%$ & $180.50 / 91.83 \%$ \\
\hline
\end{tabular}

\section{Lactatos}

Los valores de lactato son mayores en las pruebas específicas (tabla 3), especialmente en el ejercicio con un componente competitivo en el que se puede ver una diferencia significativa en los valores medios respecto al ejercicio de carrera $(E C>C C)(p>0.0320)$.

Tabla 3: Medias lactatos en pruebas continuas de carrera y específicas. Media al final de la prueba. CC: Continuo Carrera. EP: Específico Peloteo. EC: Específico Competición.

Diferencia significativa $E C>C C$. $P>0.0320$. 


\begin{tabular}{|l|c|c|c|}
\hline & CC & EP & EC \\
\hline Media final lactato & 5.00 & 5.63 & 6.63 \\
\hline
\end{tabular}

\section{Escala de Borg}

La valoración subjetiva de la fatiga ha sido inferior en los ejercicios específicos si bien no ha habido diferencia significativa. Se puede ver en la tabla 4 que el ejercicio específico de peloteo (EP) ha sido el que ha mostrado valores más bajos especialmente en comparación con el general de carrera (CC) $(p>0.0654)$.

El grado de dureza de los ejercicios fue valorado como duro, muy duro y, muy muy duro, situándose la media en la zona de duro en los tres ejercicios (15-16).

Tabla 4: Medias en la Escala de Borg en esfuerzos generales de carrera y específicos de tenis. CC: Continuo Carrera. EP: Específico Peloteo. EC: Específico Competición. No hay diferencia significativa. EP $<$ CC. $P>0.0654$.

\begin{tabular}{|l|c|c|c|}
\hline & CC & EP & EC \\
\hline $\begin{array}{l}\text { Media Escala de } \\
\text { Borg }\end{array}$ & 16.15 & 15.38 & 15.69 \\
\hline
\end{tabular}

\section{DISCUSIÓN}

Las características de las sesiones de entrenamiento o de la competición, dificultan la medición de la frecuencia cardiaca y del lactato, lo que provoca que algunos autores defiendan el uso de los sistemas subjetivos de valoración de la fatiga y la intensidad del esfuerzo para controlar la carga de trabajo realizado (Coutts y col., 2007). Sin embargo, si lo que queremos es controlar la carga real de un trabajo concreto y no solo la percepción subjetiva del jugador, deberemos asegurarnos que realmente nos aporta una información relacionada directamente con estos parámetros fisiológicos.

\section{Intensidad cardiaca y láctica}

En el estudio se ha alcanzado una alta intensidad tanto en los entrenamientos de carrera como en los que se utilizaban ejercicios de tenis, con una frecuencia cardiaca media por encima del $89 \%$ en los esfuerzos de carrera (CC), del $90 \%$ en los de peloteo (EP) y del $91 \%$ en los puntos (EC), respecto a la frecuencia cardiaca máxima individual (Anexo tabla 1). En la misma línea algunos trabajos en deportes de tipo intermitente, han mostrado también valores elevados cuando se realizaban ejercicios específicos (Hoff y Cols., 2002; Kemi y Cols., 2003; Chamari y col., 2005). En tenistas, Fernández-Fernández y col. (2011) encontraron en un trabajo específico de tenis demandas cardiacas elevadas.

En el estudio se presentan registros de frecuencia cardiaca más elevados en los trabajos específicos (EP-EC), que en los generales, con valores 
significativamente superiores en EC frente a los mostrados en CC (figura 1), siendo significativamente superior cuando existía un componente competitivo respecto a la carrera continua.

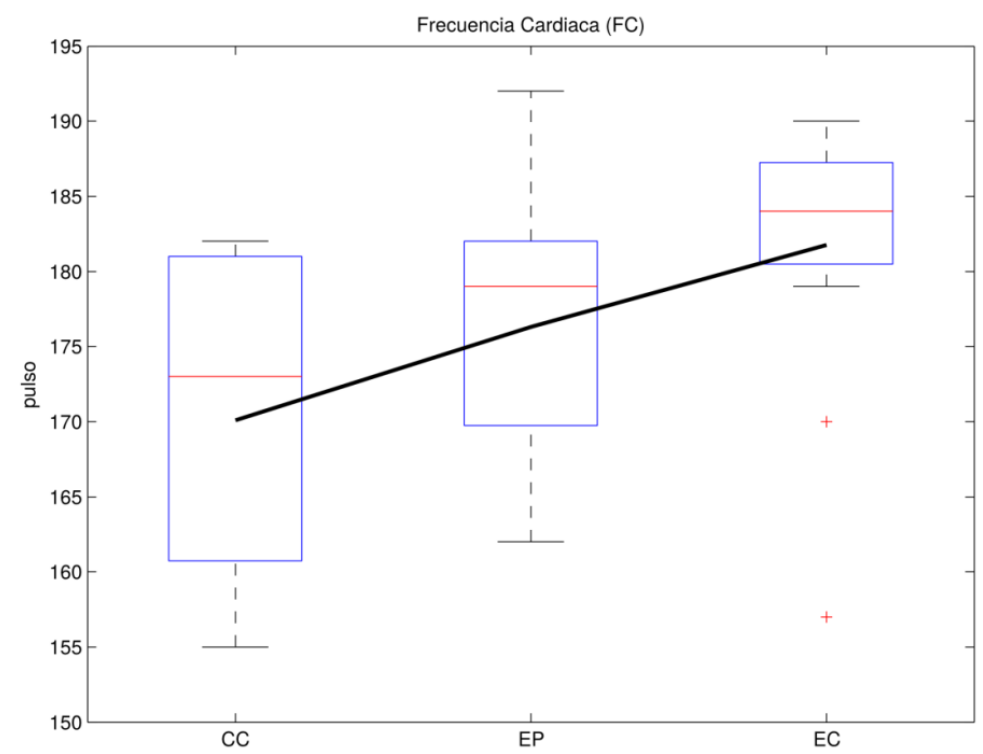

Figura 1: Distribución de la frecuencia cardiaca media en los diferentes trabajos: CC: Carrera Continua. EP: Específico Peloteo. EC: Específico Competición. La caja indica la dispersión de la muestra, definida por los percentiles 25 (límite inferior) y 75 (límite superior); la línea roja define la mediana y las líneas negras los límites inferior y superior, fuera de los cuales se considera que los valores están fuera de rango (outliers). Éstos se marcan con cruces rojas.

Como ocurre con la frecuencia cardiaca los esfuerzos de carácter específico mostraron intensidades lácticas superiores, siendo también especialmente altas cuando se realizaron mediante puntos (EC) (figura 2).

Algunos autores señalan que cuando se alcanza el consumo máximo de oxígeno, las concentraciones de lactato se aproximan a los 8-9 mmol/l (Astrand y col., 2010);en nuestro estudio estos valores fueron alcanzados principalmente en los ejercicios específicos competitivos (EC). Esta elevada concentración de lactato mediante entrenamientos específicos ha sido observada también en deportes de equipo (Hill-Hass y col., 2011).

Parece que al alcanzar con los ejercicios específicos una mayor respuesta fisiológica se permite una mayor acción sobre los procesos adaptativos relacionados con el incremento del consumo máximo de oxígeno, incidiendo de forma local sobre los tejidos implicados en el juego. La mayor variabilidad en direcciones y cambios de ritmo puede provocar, incluso en trabajos de tipo continuo, un mayor componente excéntrico y a su vez una participación mayor de las fibras rápidas, pudiendo conllevar un estímulo mixto aeróbico y anaeróbico más intenso en las fibras de tipo II. 


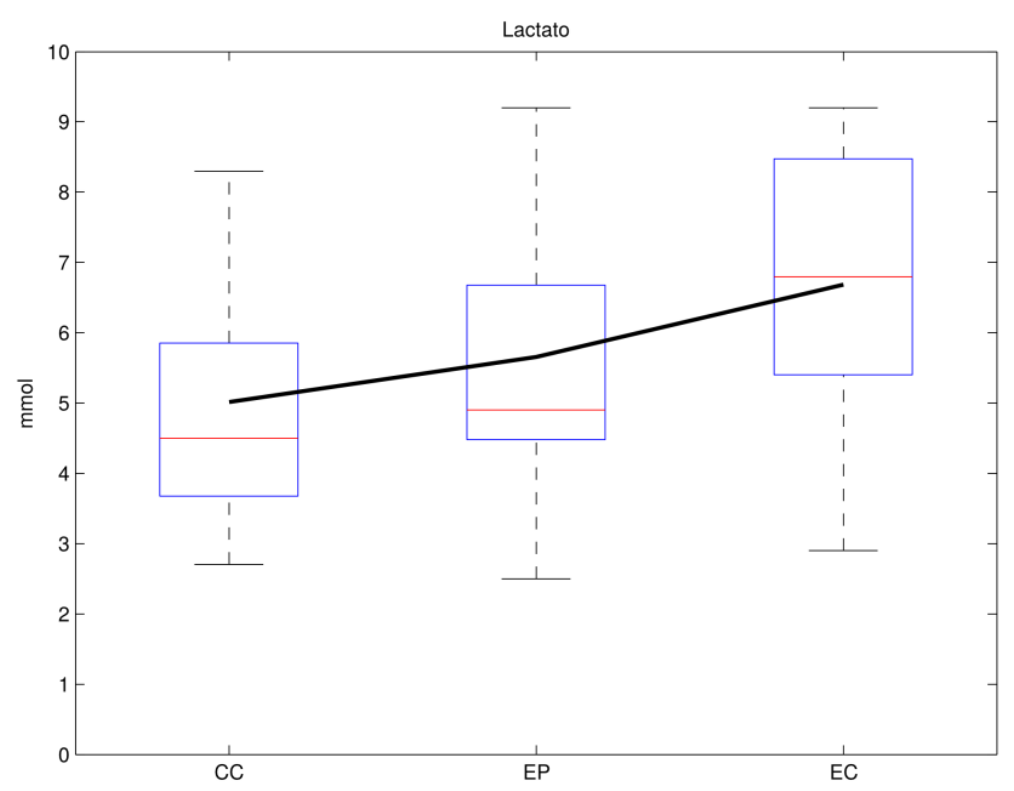

Figura 2: Distribución del lactato de los individuos para los diferentes trabajos: CC: Carrera Continua. EP: Específico Peloteo. EC: Específico Competición. La caja indica la dispersión de la muestra, definida por los percentiles 25 (límite inferior) y 75 (límite superior); la línea roja define la mediana y las líneas negras los límites inferior y superior, fuera de los cuales se considera que los valores están fuera de rango (outliers). Éstos se marcan con cruces rojas.

\section{Percepción de la fatiga y relación con la respuesta fisiológica}

Los resultados obtenidos muestran una percepción baja de la fatiga por parte de los jugadores cuando realizaron esfuerzos específicos de tenis (figura 3). No hemos encontrado diferencias estadísticamente significativas entre EC y CC en la valoración subjetiva de la fatiga a pesar de que la frecuencia cardiaca y el nivel de lactato son significativamente superiores cuando existe componente competitivo. De hecho, en ambos ejercicios específicos la valoración de la fatiga fue inferior respecto a la que se daba en la carrera continua. Reforzando esto, el ejercicio de peloteo (EP) estuvo cerca de ser significativamente inferior al de carrera continua $(C C)$ ( $>>0.0654)$, mientras que los parámetros fisiológicos medidos obtuvieron resultados más altos.

El grado de dureza de los ejercicios fue valorado en la zona de duro en los tres ejercicios (15-16). No obstante, se puede ver que los valores de frecuencia cardiaca e intensidad que se relacionan con valores de 16 son los de los ejercicios específicos en los que se dio una valoración sobre 15, y, sin embargo, en el de carrera que está más situado en 15, la percepción de la fatiga es valorada por encima de 16.

Estos datos están en la misma línea que los obtenidos por Zouhal y col. (2013) que observaron valores superiores, de frecuencia cardiaca y lactato en trabajo intermitente, en ejercicios específicos de fútbol respecto a los de carrera, siendo la percepción del grado de esfuerzo más baja en los trabajos específicos. 
Se observa una relación entre la valoración numérica, en la Escala 20 de Borg, de la fatiga y la frecuencia cardiaca, pero existe una sobrevaloración de la fatiga en esfuerzos generales de carrera y una infravaloración en los específicos de tenis. Lo que implica que es necesario tener en cuenta estas posibles diferencias a la hora de controlar la carga, valorar la intensidad, y escoger un modelo de entrenamiento $\mathrm{u}$ otro.

Hay que considerar que a pesar de haberse establecido una relación entre la intensidad fisiológica de ejercicio y la percepción subjetiva de la fatiga mostrada en la Escala de Borg (Seiler y Klerland., 2006; Weston y col., 2006; Little y Williams, 2007; Borresen y Lambert, 2008; Coquart y col., 2009; Sinclair y col., 2009), existen factores como el componente lúdico o competitivo de un ejercicio, el grado de motivación, o la mayor o menor preparación para un tipo de ejercicio, que pueden alterar dicha percepción y su relación con los parámetros fisiológicos, por lo que se debe tener precaución cuando se intente relacionar diferentes parámetros.

Parece clara la necesidad de tener en cuenta los diversos factores al usar un parámetro psicológico como la Escala de Borg; al mismo tiempo, la mayor o menor experiencia en un tipo de acción motora también puede influir en la percepción de la fatiga por parte del deportista, si bien la Escala de Borg ha sido validada en deportistas jóvenes (Impellizzeri y col., 2004). Existe una conexión lógica entre el estado anímico y las apreciaciones cognitivas englobando desde las decisiones tácticas, hasta la percepción del esfuerzo realizado (Skinner y Brewer, 2002; Liberal y García, 2011).

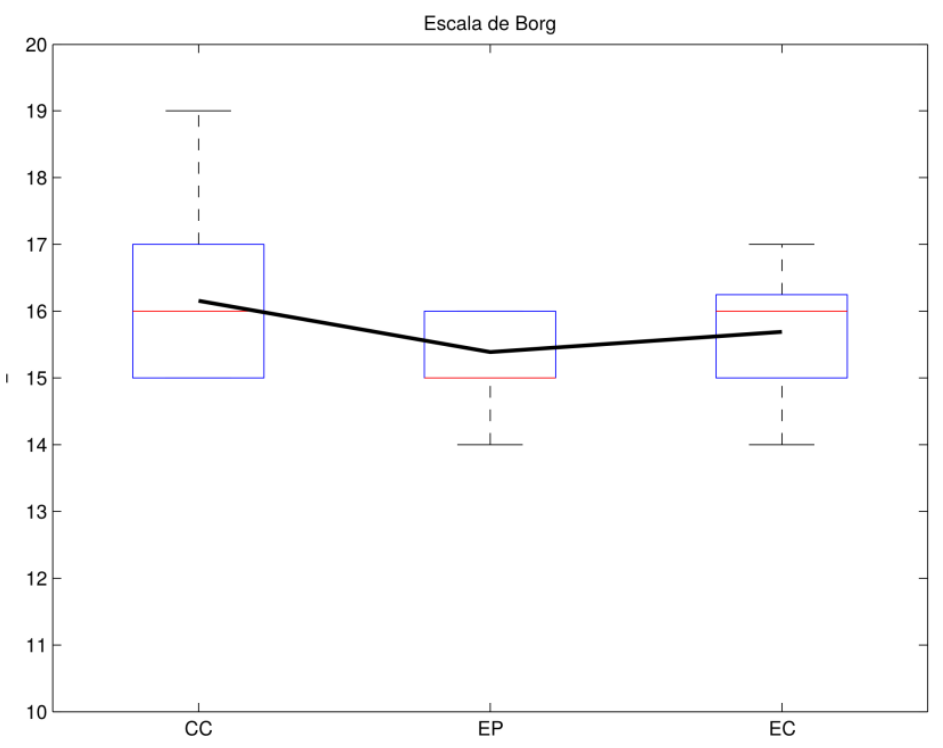

Figura 3: Distribución de la Escala de Borg de los individuos para los diferentes trabajos: CC: Carrera Continua. EP: Específico Peloteo. EC: Específico Competición. La caja indica la dispersión de la muestra, definida por los percentiles 25 (límite inferior) y 75 (límite superior); la línea roja define la mediana y las líneas negras los límites inferior y superior, fuera de los cuales se considera que los valores están fuera de rango (outliers). Éstos se marcan con cruces rojas. 


\section{CONCLUSIONES}

Podemos concluir que los entrenamientos específicos de tenis utilizados tuvieron intensidades elevadas e incluso mayores que los de carrera, permitiendo un estímulo adecuado de tipo mixto aeróbico-anaeróbico mientras se trabajaba situaciones de juego real. Esto fue especialmente significativo cuando se introducía el elemento competitivo. No obstante, creemos que el entrenamiento mediante puntos no sería el más adecuado, por su dificultad de control, cuando el objetivo es entrenar con una menor intensidad.

De nuestra investigación se desprende que en los entrenamientos específicos de tenis, la percepción subjetiva de la fatiga, y por lo tanto la exigencia del esfuerzo, puede resultar inferior a la que cabría esperar en función de la intensidad fisiológica alcanzada, para este grupo. Por tanto, hay que tener en cuenta la infravaloración que se puede hacer de la fatiga y grado de intensidad de un trabajo cuando utilizamos la Escala de Borg en la competición y el entrenamiento mediante ejercicios específicos de tenis.

\section{REFERENCIAS BIBLIOGRÁFICAS}

1. Alexiou H, Coutts AJ. (2008). A comparison of methods used for quantifying internal training load in women soccer players. Int J Sports Physiol Perform. 3:320-330. https://doi.org/10.1123/ijspp.3.3.320

2. Algroy EA, Hetelid KJ, Seiler S, Stray Pedersen JI. (2011). Quantifying training intensity distribution in a group of Norwegian Professional soccer players. Int J Sports Physiol Perform. 6:70-81. https://doi.org/10.1123/ijspp.6.1.70

3. Astrand PO, Rodahl K, Hans AD, Sigmund BS. Manual de fisiología del ejercicio. Paidotribo. Barcelona. 2010.

4. Borg E, Kaijser L. (2006). A comparison between three rating scales for perceived exertion and two different work tests. Scand J Med Sci Sports. 16:57-69. https://doi.org/10.1111/j.1600-0838.2005.00448.x

5. Borresen J, Lambert Ml. (2008). Quantifying training load: A comparison of subjective and objective methods. Int $\mathrm{J}$ Sport Physiol Perf. 3:16-30. https://doi.org/10.1123/ijspp.3.1.16

6. Calahorro F, Torres-Luque G, Lara AJ, Zagalaz ML. (2011). Parameters related to the competition's physical training. J Sport Healt Res. 3:5-7.

7. Coquart J, Legrand R, Robin S, Duhamel A, Matran R, Garcin M. (2009). Influence of successive bouts of fatiguing exercise on perceptual and physiological markers during an incremental exercise test. Psychophysiology. 46:209-216. https://doi.org/10.1111/j.1469-8986.2008.00717.x

8. Coutts AJ, Rampininib E, Marcorac SM, Castagna C, Impellizeri FM. (2007). Heart rate and blood lactate correlates of perceived exertion during smallsided soccer games. J Sci Med Sport. 12:79-84. https://doi.org/10.1016/i.jsams.2007.08.005

9. Cuadrado-Reyes J, Chirosa LJ, Chirosa I, Martín-Tamayo I, Aguilar-Martínez D. (2012). La percepción subjetiva del esfuerzo para el control de la carga de entrenamiento en una temporada en un equipo de balonmano. RevPsicolDeport. 21:331-339. 
10. Chamari K, Hachana Y, Kaouech F, Jeddi R, Moussa-Chamari I, Wisløff U. (2005). Endurance training and testing with the ball in young elite soccer $\begin{array}{lllll}\text { players. } & \mathrm{Br} & \mathrm{J} & \text { Sports } & \text { Med. }\end{array}$ https://doi.org/10.1136/bjsm.2003.009985

11. Faulkner J, Parfitt G, Eston R. (2007). Prediction of maximal oxygen uptake from the ratings of perceived exertion and heart rate during a perceptuallyregulated sub-maximal exercise test in active and sedentary participants. Eur J Appl Physiol. 101:397-407. https://doi.org/10.1007/s00421-007-0508-6

12. Fernandez-Fernandez J, Sanz-Rivas D, Sanchez-Muñoz C, de la Aleja Tellez JG, Buchheit M, Mendez-Villanueva A. (2011). Physiological Responses to On-Court vs Running Interval Training in Competitive Tennis Players. J Sports Sci Med. 10:540-5.

13. Hill-Haas SV, Rowsell GJ, Dawson BT, Coutts AJ. (2009). Acute physiological responses and time-motion characteristics of two small-sided training regimes in youth soccer players. J Strength Cond Res. 23:111-115. https://doi.org/10.1519/JSC.0b013e31818efc1a

14. Hill-Hass SV, Dawson B, Impellizzeri FM, Coutts AJ. (2011). Physiology of small-sided games training in football: a systematic review. Sports Med. 41:199-220. https://doi.org/10.2165/11539740-000000000-00000

15. Hoff J, Wisloff U, Engen LC, Kemi OJ, Helgerud J. (2002). Soccer specific aerobic endurance training. $\mathrm{Br} J$ Sports Med. 36:218-221. https://doi.org/10.1136/bjsm.36.3.218

16. Impellizzeri, F., Rampinini, E., Coutts, A., Sassi, A. y Marcora, S. (2004). Use of RPE-Based Training Load in Soccer. Medicine and science in sports and exercise.36

1042-1047. https://doi.org/10.1249/01.MSS.0000128199.23901.2F

17. Kemi OJ, Hoff J, Engen LC, Helgerud J, Wisloff U. (2003). Soccer specific testing of maximal oxygen uptake. J Sports Med Phys Fit. 43:139-144.

18. Krustrup P, Mohr M, Steensberg A, et al. (2006). Muscle and Blood Metabolites during a Soccer Game: Implications of Sprint Performance. Med Sci Sports Exerc. 38:1165-1174. https://doi.org/10.1249/01.mss.0000222845.89262.cd

19. Liberal García R, García Más A. Percepción de dolor y fatiga en relación con el estado de ánimo. Cuad Psicol Deporte. 2011; 11:93-106.

20. Little Th, Williams AG. Measures of exercise intensity during soccer training drills with professional soccer players. J Strength Cond Res. 2007; 21:367371. https://doi.org/10.1519/00124278-200705000-00013

21. Méndez-Villanueva A, Fernández-Fernández J, Bishop D. (2007). Exercise induced homeostatic perturbations provoked by singles tennis match play with reference to development of fatigue. $\mathrm{Br} J$ SportsMed. 41:717-722. https://doi.org/10.1136/bjsm.2007.037259

22. Ostojic SM, Mazic S. (2002). Effects of a carbohydrate-electrolyte drink on specific soccer tests and performance. J Sports Sci Med. 2:47-53.

23. Potteiger JA. Entrenamiento de la resistencia aeróbica. (2007). En: Baechle, ThR; Earle, RW. Principios del Entrenamiento de la Fuerza y del Acondicionamiento Físico. Panamericana.

24. Seiler K, Klerland G. (2006). Quantifying training intensity distribution in elite endurance athletes: is there evidence for an optimal distribution? 
Scandinavian J Med \&Sci Sports. 16:49-56. https://doi.org/10.1111/j.16000838.2004.00418.x

25. Sinclair W, Kerr R, Spinks W, Leicht A. (2009). Blood lactate, heart rate and rating of perceived exertion responses of elite surf lifesavers to highperformance competition. J Sci Med Sport. 12:101-106. https://doi.org/10.1016/i.jsams.2007.10.008

26. Skinner N, Brewer N. (2002). The dynamics of threat and challenge appraisals prior to stressful achievement events. J Personality Social Psychol. 83:678692. https://doi.org/10.1037//0022-3514.83.3.678

27. Torres-Luque G, Sánchez-Pay A, Belmonte MJB, Ramón MM. (2011). Functional aspects of competitive tennis. J Hum Sport Exerc. 6:528-539. https://doi.org/10.4100/jhse.2011.63.07

28. Weston M, Bird S, Helsen W, Nevill A, Castagna C. (2006). The effect of match standard and referee experience on the objective and subjective match workload of English Premier League referees. J SciMed Sport. 9:256-262. https://doi.org/10.1016/i.jsams.2006.03.022

29. Zouhal H, LeMoal E, Wong DP, BenOunis O, Castagna C, Duluc C, Owen AL, Drust B. (2013). Physiological Responses of General vs. Specific Aerobic Endurance Exercises in Soccer. As J Sports Med. 4:212-220. https://doi.org/10.5812/asjsm.34285

Número de citas totales / Total references: 29 (100\%)

Número de citas propias de las revista / Número de citas propias de la revista / Journal's own references: $0(0 \%)$

Rev.int.med.cienc.act.fís.deporte- vol. 19 - número 75 - ISSN: 1577-0354

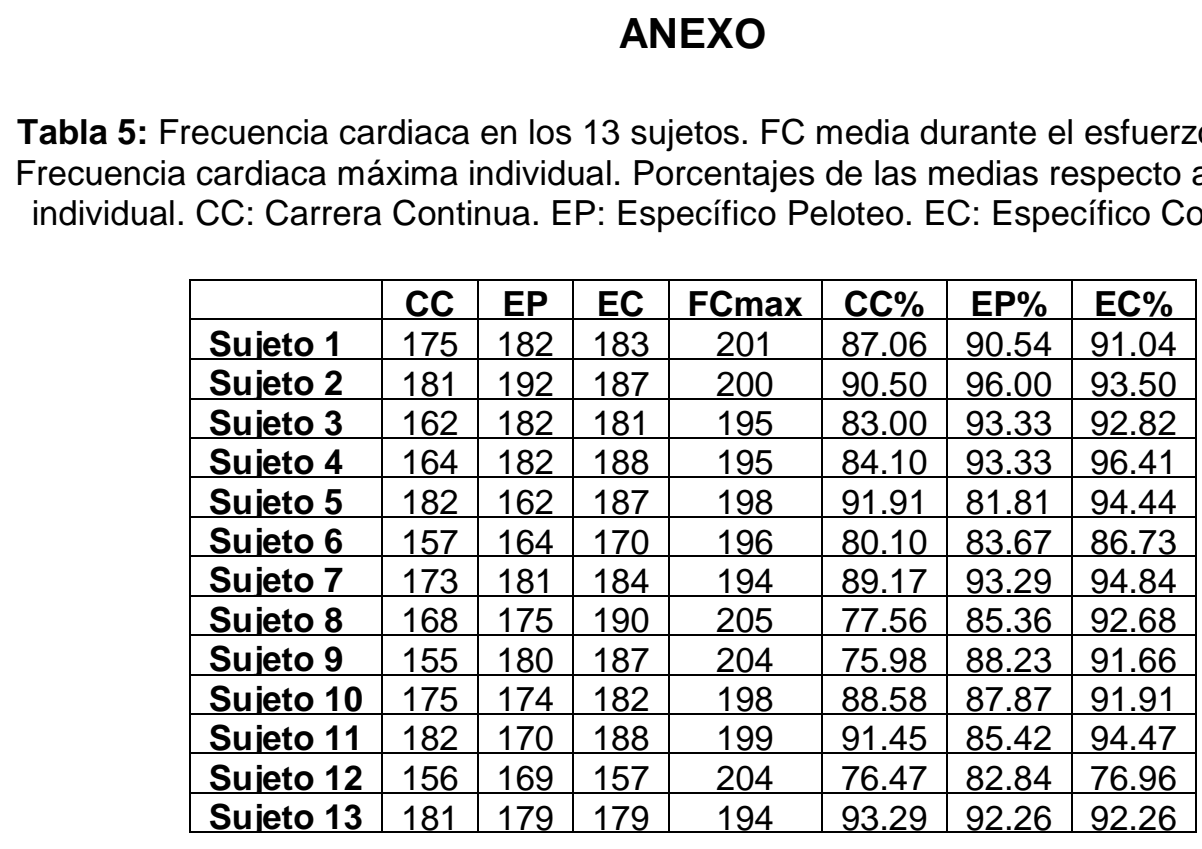


Tabla 6: Comparación (izquierda) y significación estadística (derecha), dada por el p-valor, de las medias de frecuencia cardiaca de las pruebas: CC: Carrera Continua. EP: Específico

Peloteo. EC: Específico Competición. La hipótesis nula (las medias son iguales) se rechazará $(\mathrm{HO}=-1$ ó $\mathrm{HO}=1)$ con una significación del $95 \%$ si el $\mathrm{p}$-valor es menor que 0.05 . El signo asignado a $\mathrm{H} 0$ indica si la media en la columna es mayor $(-1)$ o menor $(+1)$ que en la fila.

\begin{tabular}{|c|c|c|c|c|c|c|}
\hline & CC & EP & EC & CC & EP & EC \\
\hline CC & 0 & 0 & -1 & 1.0000 & 0.1044 & $\mathbf{0 . 0 0 5 4}$ \\
\hline EP & 0 & 0 & 0 & 0.1044 & 1.0000 & 0.1246 \\
\hline EC & $\mathbf{1}$ & 0 & 0 & $\mathbf{0 . 0 0 5 4}$ & 0.1246 & 1.0000 \\
\hline
\end{tabular}

Tabla 7: Comparación (izquierda) y significación estadística (derecha), dada por el p-valor, de las medias de frecuencia cardiaca relativa respecto a la frecuencia cardiaca máxima individual de las pruebas: CC: Carrera Continua. EP: Específico Peloteo. EC: Específico Competición. La hipótesis nula (las medias son iguales) se rechazará ( $\mathrm{HO}=-1$ ó $\mathrm{HO}=1$ ) con una significación del $95 \%$ si el p-valor es menor que 0.05 . El signo asignado a $\mathrm{H} 0$ indica si la media en la columna es mayor $(-1)$ o menor $(+1)$ que en la fila.

\begin{tabular}{|c|c|c|c|c|c|c|}
\hline & CC & EP & EC & CC & EP & EC \\
\hline CC & 0 & 0 & $\mathbf{- 1}$ & 1.0000 & 0.1458 & $\mathbf{0 . 0 1 0 8}$ \\
\hline EP & 0 & 0 & 0 & 0.1458 & 1.0000 & 0.1246 \\
\hline EC & $\mathbf{1}$ & 0 & 0 & $\mathbf{0 . 0 1 0 8}$ & 0.1458 & 1.0000 \\
\hline
\end{tabular}

Tabla 8: Lactato en los 13 sujetos. CC: Continuo Carrera. EP: Específico Peloteo. EC: Específico Competición.

\begin{tabular}{|l|l|l|l|}
\hline & CC & EP & EC \\
\hline Suieto 1 & 8.3 & 5.5 & 4.1 \\
\hline Suieto 2 & 6.6 & 9.1 & 5.7 \\
\hline Suieto 3 & 4.5 & 2.5 & 2.9 \\
\hline Suieto 4 & 5.2 & 4.4 & 6.6 \\
\hline Suieto 5 & 5.1 & 4.5 & 9.2 \\
\hline Suieto 6 & 3.1 & 4.1 & 5.5 \\
\hline Suieto 7 & 4.5 & 6.3 & 7.1 \\
\hline Suieto 8 & 3.3 & 4.7 & 8.4 \\
\hline Suieto 9 & 2.7 & 7.8 & 9.1 \\
\hline Suieto 10 & 4.4 & 4.9 & 6.8 \\
\hline Suieto 11 & 5.6 & 4.7 & 7.7 \\
\hline Suieto 12 & 3.8 & 5.8 & 5.1 \\
\hline Suieto 13 & 8.1 & 9.2 & 8.7 \\
\hline
\end{tabular}

Tabla 9: Comparación (izquierda) y significación estadística (derecha), dada por el p-valor, de las medias de lactato al final de las pruebas. CC: Carrera Continua. EP: Específico Peloteo. EC: Específico Competición. La hipótesis nula (las medias son iguales) se rechazará ( $\mathrm{H} 0=-1$ ó $\mathrm{HO}=1$ ) con una significación del $95 \%$ si el p-valor es menor que 0.05 . El signo asignado a HO indica si la media en la columna es mayor $(-1)$ o menor $(+1)$ que en la fila.

\begin{tabular}{|c|c|c|c|c|c|c|}
\hline & CC & EP & EC & CC & EP & EC \\
\hline CC & 0 & 0 & $\mathbf{- 1}$ & 1.0000 & 0.3949 & $\mathbf{0 . 0 3 2 0}$ \\
\hline EP & 0 & 0 & 0 & 0.3949 & 1.0000 & 0.1954 \\
\hline EC & $\mathbf{1}$ & 0 & 0 & $\mathbf{0 , 0 3 2 0}$ & 0.1954 & 1.0000 \\
\hline
\end{tabular}

Tabla 10: Escala de Borg en los 13 sujetos. CC: Continuo Carrera. EP: Específico Peloteo. EC: Específico Competición. 


\begin{tabular}{|l|c|c|c|}
\hline & CC & EP & EC \\
\hline Suieto 1 & 15 & 16 & 17 \\
\hline Suieto 2 & 17 & 15 & 15 \\
\hline Suieto 3 & 19 & 16 & 16 \\
\hline Suieto 4 & 15 & 15 & 17 \\
\hline Suieto 5 & 17 & 14 & 15 \\
\hline Suieto 6 & 15 & 15 & 15 \\
\hline Suieto 7 & 15 & 16 & 16 \\
\hline Suieto 8 & 17 & 16 & 16 \\
\hline Suieto 9 & 15 & 15 & 15 \\
\hline Suieto 10 & 17 & 16 & 17 \\
\hline Suieto 11 & 16 & 16 & 16 \\
\hline Suieto 12 & 15 & 15 & 15 \\
\hline Suieto 13 & 17 & 15 & 14 \\
\hline
\end{tabular}

Tabla 11: Comparación (izquierda) y significación estadística (derecha), dada por el p-valor, de las medias de Escala de Borg: Carrera Continua. EP: Específico Peloteo. EC: Específico Competición. La hipótesis nula (las medias son iguales) se rechazará $(H 0=-1$ ó $H 0=1)$ con una significación del $95 \%$ si el p-valor es menor que 0.05 . El signo asignado a H0 indica si la media en la columna es mayor $(-1)$ o menor $(+1)$ que en la fila.

\begin{tabular}{|c|c|c|c|c|c|c|}
\hline & CC & EP & EC & CC & EP & EC \\
\hline CC & 0 & 0 & 0 & 1.0000 & 0.0654 & 0.3067 \\
\hline EP & 0 & 0 & 0 & 0.0654 & 1.0000 & 0.3440 \\
\hline EC & 0 & 0 & 0 & 0.3067 & 0.3440 & 1.0000 \\
\hline
\end{tabular}

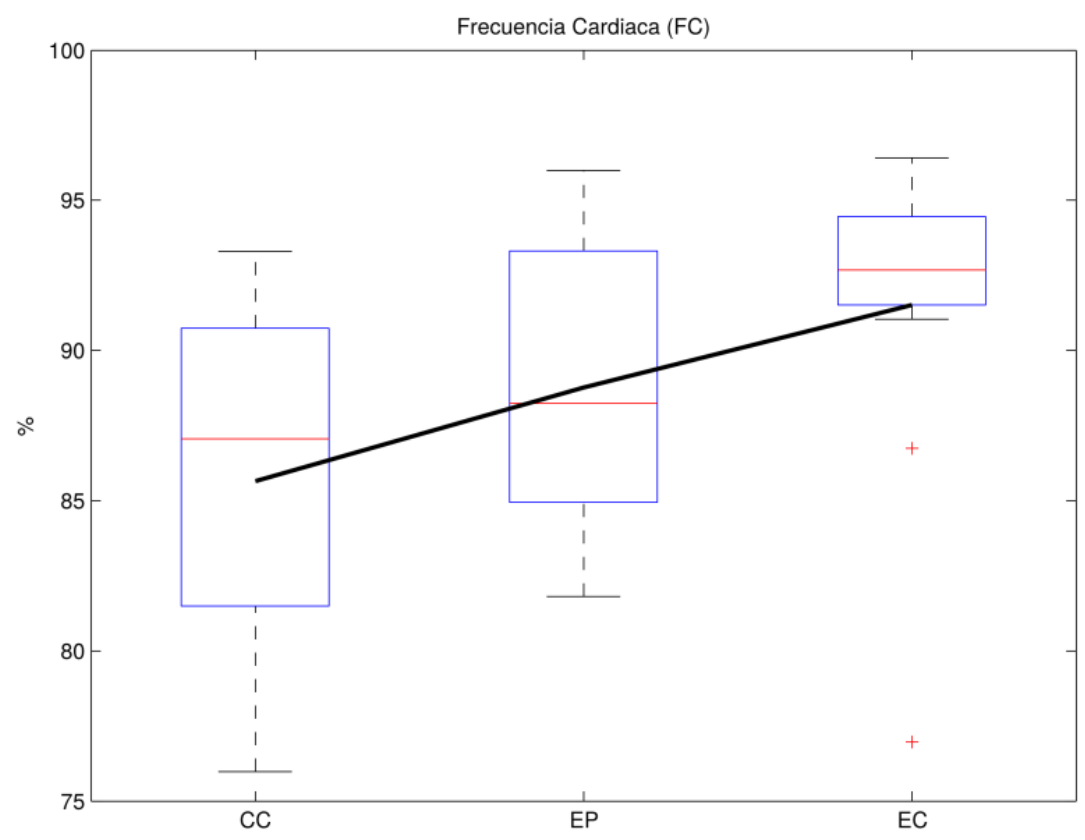

Figura 4: Distribución de la frecuencia cardiaca media relativo a la frecuencia máxima individual en los diferentes trabajos: CC: Carrera Continua. EP: Esfuerzo Peloteo. EC:

Esfuerzos Competición. La caja indica la dispersión de la muestra, definida por los percentiles 25 (límite inferior) y 75 (límite superior); la línea roja define la mediana y las líneas negras los límites inferior y superior, fuera de los cuales se considera que los valores están fuera de rango (outliers). Éstos se marcan con cruces rojas. 
Rev.int.med.cienc.act.fís.deporte- vol. 19 - número 75 - ISSN: 1577-0354

Rev.int.med.cienc.act.fís.deporte- vol. 19 - número 75 - ISSN: 1577-0354 